\title{
Thalamocortical Connections and Executive Function in Pediatric Temporal and Frontal Lobe Epilepsy
}

\author{
(D) N. Law, (D) M.L. Smith, and (DE. Widjaja
}

\begin{abstract}
BACKGROUND AND PURPOSE: Largely accepted in the literature is the role the interconnections between the thalamus and cortex play in generalized epilepsy. However, thalamocortical involvement is less understood in focal epilepsy in terms of the effect of seizures on thalamocortical circuitry in the developing brain and subsequent cognitive outcome. We investigated thalamocortical pathway microstructure in pediatric frontal lobe epilepsy and temporal lobe epilepsy and examined the associations between pathway microstructure and measures of executive function.
\end{abstract}

MATERIALS AND METHODS: We examined thalamocortical connections in 24 children with frontal lobe epilepsy, 17 patients with temporal lobe epilepsy, and 25 healthy children using DTI. We investigated several executive function measures in patients and controls, which were distilled into latent executive function components to compare among groups, and the associations between measures of thalamocortical microstructure and executive function.

RESULTS: We found no differences in thalamocortical pathway microstructure between the groups, but aspects of executive function (mental flexibility/inhibition/shifting) were impaired in the frontal lobe epilepsy group compared with controls. In patients with frontal lobe epilepsy, younger age at seizure onset and a greater number of antiepileptic drugs were associated with DTI indices indicative of damaged/less developed thalamocortical pathways. In patients with temporal lobe epilepsy, poorer performance on all measures of executive function was associated with DTI indices reflective of damaged/less developed pathways.

CONCLUSIONS: Our results give insight into vulnerable neural networks in pediatric focal epilepsy and suggest thalamocortical pathway damage as a potential mechanism of executive function impairment in temporal lobe epilepsy but not frontal lobe epilepsy. Identifying structure-function relations can help inform how we measure functional and cognitive/behavioral outcomes in these populations.

ABBREVIATIONS: AEDs = antiepileptic drugs; CPT3 = Conners' Continuous Performance Task, 3rd ed; D-KEFS = Delis-Kaplan Executive Function System; EF = executive function; $\mathrm{FLE}$ = frontal lobe epilepsy; $\mathrm{HC}=$ healthy controls; $\mathrm{KMO}=$ Kaiser-Meyer-Olkin Measure of Sampling Adequacy; MANOVA = multivariate analysis of variance; $\mathrm{MD}=$ mean diffusivity; $\mathrm{PCA}=$ principal components analysis; $\mathrm{RD}=$ radial diffusivity; $\mathrm{TLE}$ = temporal lobe epilepsy

S tructural and functional alterations of the brain, extending beyond the region of epileptogenic focus, may result from seizure-induced cellular changes in epilepsy. ${ }^{1}$ Thalamocortical connections are involved in the generation and propagation of

Received October 9, 2017; accepted after revision April 11, 2018.

From the Department of Psychology (N.L., M.L.S.), University of Toronto, Toronto, Ontario, Canada; and Department of Psychology (N.L., M.L.S.), Program in Neurosciences and Mental Health (N.L., M.L.S.), Diagnostic Imaging (E.W.), and Division of Neurology (E.W.), Hospital for Sick Children, Toronto, Ontario, Canada.

This research was conducted with the support of EpLink - The Epilepsy Research Program of the Ontario Brain Institute (OBI). The OBI is an independent nonprofit corporation, funded partially by the Ontario government. The opinions, results and conclusions are those of the authors and no endorsement by the Ontario Brain Institute is intended or should be inferred.

Please address correspondence to Elysa Widjaja, MD, 555 University Ave, Toronto, ON, Canada, M5G 1X8; e-mail: elysa.widjaja@sickkids.ca

三 Indicates article with supplemental on-line tables.

http://dx.doi.org/10.3174/ajnr.A5691 typical spike and wave seizures in generalized epilepsy. ${ }^{2}$ In idiopathic generalized epilepsy, abnormal thalamocortical reciprocal connections underlie epileptogenic activity via large-scale synchronization of thalamocortical loops ${ }^{3}$ or via thalamic propagation to cortical networks, ${ }^{4}$ with the thalamus acting as an extension of the cortical epileptic network during seizures. ${ }^{5}$ Thalamocortical connections have been investigated in adults with juvenile myoclonic epilepsy ${ }^{6}$ and idiopathic generalized epilepsy. ${ }^{7}$ Reduced white matter integrity of the anterior thalamus and prefrontal cortex was found in adults with juvenile myoclonic epilepsy compared with healthy controls (HC). ${ }^{6}$ In contrast, functional but not structural differences were found for thalamofrontal pathways in adults with idiopathic generalized epilepsy versus controls. ${ }^{7}$

Thalamocortical connections have been implicated in wakefulness/arousal and attention/executive function (EF), among other functions. Damage to these connections has been docu- 
mented in children with cerebellar brain tumors and developmental dyslexia and has been linked with working memory deficits $^{8}$ and reading ability, ${ }^{9}$ respectively. It is not surprising then that structural and functional alterations in these pathways have been proposed to underlie functional deficits in generalized epilepsy. ${ }^{10}$ Impairments in attention/EF have been documented in generalized epilepsy, ${ }^{10}$ which, in turn, have been associated with disruption of thalamic and frontal circuits. ${ }^{11}$ However, thalamocortical involvement is less understood in focal epilepsy.

Children with frontal lobe epilepsy (FLE) have deficits in EF, including planning and impulse control, ${ }^{12}$ verbal fluency, and mental flexibility. ${ }^{13}$ There is also evidence of EF impairment in children with temporal lobe epilepsy (TLE), ${ }^{14}$ though EF deficits may be more pronounced in FLE. ${ }^{12,15-17}$ It is possible that a common neural substrate-thalamocortical connections-could be affected in FLE and to a lesser extent in TLE, and this compromise, in turn, may influence EF.

Widespread reduced functional and structural connectivity has been documented in children with FLE, such as reduced frontal network connectivity, ${ }^{18}$ and multilobar white matter abnormalities, including frontal connections. ${ }^{19}$ Reduced connectivity within the frontal network was related to performance in EF in children with FLE. ${ }^{18}$ Cognitively impaired children with FLE showed the greatest white matter compromise compared with cognitively unimpaired children with FLE and HC. ${ }^{20}$ Several studies have documented structural abnormalities in association fibers such as the superior longitudinal fasciculus, inferior frontooccipital fasciculus, uncinate fasciculus, cingulum, forceps minor, and corpus callosum in patients with focal epilepsy. ${ }^{21-24}$ Abnormality in the uncinate fasciculus has been shown to correlate with EF in patients with TLE. ${ }^{25}$ Additionally, abnormal association fibers have been shown to correlate with EF in other neurologic disorders. ${ }^{26,27}$ For the current study, we chose to examine connections between the thalamus and ipsilateral frontal lobe for the following reasons: 1) Thalamocortical fibers have been implicated in both adult and pediatric generalized epilepsy, 2) less is known about the role of thalamocortical pathways in focal epilepsy, and 3) thalamocortical connections and their microstructure have been previously linked to aspects of EF in other pediatric populations. ${ }^{8}$

We examined thalamocortical pathways in children with FLE, TLE, and HC; measures of EF; and the associations between pathway microstructure and EF to determine whether thalamocortical pathways are a potential mechanism of EF deficits in focal epilepsy. We hypothesized that altered thalamocortical microstructure would be observed in both FLE and TLE, with patients with FLE showing the greatest compromise to the pathways. Furthermore, multiple components of EF would be impaired in children with FLE compared with those with TLE and HC. Last, measures of thalamocortical microstructure would be correlated with EF.

\section{MATERIALS AND METHODS \\ Participants}

Participants included 24 children with FLE, 17 with TLE, and 25 HC. All patients had nonlesional etiology, and epileptogenic focus was defined on the basis of ictal video electroencephalography. The protocol for this study was approved by the Research Ethics Board, and informed consent was obtained. Table 1 contains de-
Table 1: Demographic and medical variables for the HC, FLE, and TLE groups

\begin{tabular}{|c|c|c|c|}
\hline & $\begin{array}{c}\text { FLE } \\
(n=24)\end{array}$ & $\begin{array}{c}\text { TLE } \\
(n=17)\end{array}$ & $\begin{array}{c}\text { HC } \\
(n=25)\end{array}$ \\
\hline Sex (male/female) & $10: 14$ & $8: 9$ & 14:11 \\
\hline \multicolumn{4}{|l|}{ Age (yr) } \\
\hline Mean (SD) & $13.10(3.21)$ & $14.54(2.65)$ & $13.95(3.20)$ \\
\hline Range & $6.96-16.96$ & $10.11-19.08$ & $6.97-18.76$ \\
\hline Handedness (right/left) & $20 / 4$ & $16 / 1$ & $22 / 3$ \\
\hline \multicolumn{4}{|l|}{ Age at seizure onset (yr) } \\
\hline Mean (SD) & $7.79(4.01)$ & $8.75(3.76)$ & - \\
\hline Range & $1.00-14.40$ & $1.30-15.00$ & - \\
\hline \multicolumn{4}{|l|}{ Duration of epilepsy (yr) } \\
\hline Mean (SD) & $5.20(2.93)$ & $4.90(3.62)$ & - \\
\hline Range & $1.70-12.00$ & $.33-12.00$ & - \\
\hline \multicolumn{4}{|l|}{ No. of AEDs } \\
\hline Mean (SD) & $2.00(.659)$ & $1.94(.748)$ & - \\
\hline Range & $1-3$ & $1-3$ & - \\
\hline \multicolumn{4}{|l|}{$\begin{array}{l}\text { Laterality of epileptogenic } \\
\text { focus }\end{array}$} \\
\hline Left & 13 & 10 & - \\
\hline Right & 9 & 4 & - \\
\hline Bilateral & 2 & 3 & - \\
\hline \multicolumn{4}{|l|}{$\begin{array}{l}\text { Region of epileptogenic } \\
\text { focus (TLE) }\end{array}$} \\
\hline Mesial & - & 3 & - \\
\hline Lateral & - & 12 & - \\
\hline Both mesial and lateral & - & 2 & - \\
\hline \multicolumn{4}{|l|}{$\begin{array}{l}\text { Region of epileptogenic } \\
\text { focus (FLE) }\end{array}$} \\
\hline Prefrontal & 2 & - & - \\
\hline Inferior frontal & 1 & - & - \\
\hline Frontal-central & 9 & - & - \\
\hline Supplementary motor area & 3 & - & - \\
\hline Unable to localize precisely & 9 & - & - \\
\hline
\end{tabular}

mographic and medical variables. There were no differences among the groups for sex $\left[\chi_{(2)}^{2}=1.026, P=.599\right]$, age at study $[F(2,63)=1.138, P=.327]$, and handedness $\left[\chi_{(2)}^{2}=1.087, P=\right.$ $.581]$. The epilepsy groups were comparable for age at seizure onset $[F(1,39)=0.572, P=.454]$, duration of epilepsy $[F(1,39)=$ $0.084, P=.774]$, laterality of epileptogenic focus $\left[\chi_{(2)}^{2}=1.359\right.$, $P=.507]$, and number of antiepileptic drugs (AEDs) $[F(1,39)=$ $0.071, P=.791]$.

\section{Neuroimaging Protocol}

MR imaging was performed on an Achieva 3T scanner (Philips Healthcare, Best, the Netherlands) using an 8-channel phased array head coil in all patients and controls. The imaging consisted of axial and coronal FLAIR, proton-density/T2, volumetric T1, and DTI $(\mathrm{TR} / \mathrm{TE}=10,000 / 60 \mathrm{~ms}$, slice thickness $=2 \mathrm{~mm}, \mathrm{FOV}=22$ $\mathrm{cm}$, matrix $=112 \times 112, \mathrm{NEX}=1$, sensitivity encoding $=1$, $b=1000 \mathrm{~s} / \mathrm{mm}^{2}, 32$ noncollinear directions). The DTI indices and maps (eg, eigenvectors, fractional anisotropy, mean diffusivity [MD], axial diffusivity, and radial diffusivity $[R D]$ ) were calculated using the FMRIB Software Library (FSL; http://www.fmrib. ox.ac.uk/fsl $)^{28}$ to serve as a basis for probabilistic tractography.

ROI Placement and Standardization for DTI Tractography To provide a standardized protocol for ROI placement, we drew all ROIs on axial sections of the zero diffusion-weighted (b0) images of 10 randomly selected HC. The following 4 ROIs were 


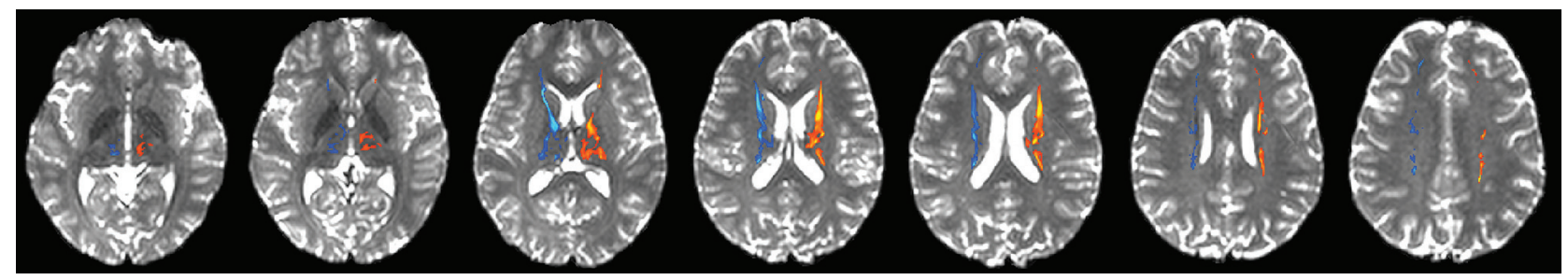

FIG 1. Thalamocortical pathways connecting the thalamus with the frontal cortex in a healthy brain. The left thalamocortical pathway is depicted in red/yellow, and the right thalamocortical pathway is depicted in dark/light blue. Axial sections are presented as the tracts progress dorsally from the thalamus to the frontal lobe (left to right).

produced for all 10 individuals using FSL ${ }^{29}$ : left and right frontal hemispheric white matter (ie, the frontal lobe was masked in its entirety for each hemisphere) and left and right thalamus (ie, masks were placed over the entirety of the thalamus of each hemisphere). The 10 sets of individually delineated ROIs were then combined into a single composite volume and registered to a brain template using Automated Image Registration ${ }^{30}$ to produce template ROIs. The resultant template ROIs were registered onto each participant's b0 images using Automated Image Registration. ${ }^{30}$ ROIs were brought into the native space for each participant, visually examined, and, if necessary, manually refined so that they covered only the appropriate region.

\section{DTI Probabilistic Tractography and Pathway Segmentation}

Using the FMRIB Diffusion Toolbox (http://fsl.fmrib.ox.ac.uk/ fsl/fslwiki/FDT), ${ }^{29}$ we completed probabilistic tractography to delineate bilateral thalamocortical white matter pathways connecting the left or right thalamus with the ipsilateral frontal cortex and to examine the microstructure of pathways. Within each hemisphere, to delineate the thalamocortical pathway, we used the thalamus ROI as the seed point and the ipsilateral frontal hemisphere ROI as the waypoint. The threshold was set to $10 \%$ for all pathways and was edited, as appropriate, to eliminate erroneous streamlines. Means and SDs for DTI indices were calculated for each pathway.

\section{Measures of EF}

Delis-Kaplan Executive Function System. The Verbal Fluency, Color-Word Interference, and Sorting subtests of the Delis-Kaplan Executive Function System (D-KEFS) ${ }^{31}$ measured generative naming, cognitive efficiency, response inhibition, mental flexibility, and planning/problem solving (see On-line Table 1 for test descriptions). Raw scores were converted to norm-referenced scaled scores before statistical analysis.

Conners' Continuous Performance Task, 3rd ed. We used the Conners' Continuous Performance Task, 3rd ed (CPT3), ${ }^{32}$ a computer-based task, to measure sustained attention. Omission errors (ie, failure to respond to target letters, a measure of inattention) T-scores were obtained and converted to scaled scores before analysis.

\section{Statistics/Analytic Approach}

The FLE and TLE groups were divided into 4 groups: left FLE ( $n=$ $13)$, right FLE $(n=9)$, left TLE $(n=10)$, and right TLE $(n=4)$, and group means were compared with HC ( $n=25)$ using multivariate analysis of variance (MANOVA). All participants with bilateral seizure focus were omitted from these analyses $(n=5)$. We performed 2 MANOVAs on DTI indices for the left and right thalamocortical pathways separately, with group as the betweensubject variable. Planned tests of simple effects were used to examine the a priori hypothesis that the FLE group would show compromise in white matter microstructure within the thalamocortical pathways relative to the TLE and HC groups.

Next, we used principal component analysis (PCA) to reduce our measures of EF into component factors that represented underlying/latent EFs across our entire sample. As part of a dataquality examination process, we used the Kaiser-Meyer-Olkin Measure of Sampling Adequacy (KMO) and the Bartlett Test of Sphericity to determine the suitability of our data for being entered into PCA for factor/structure detection. A varimax rotation was used. The factor solution was based on those components with eigenvalues of $>1.0$ and the interpretability of the components (eg, loadings of $>.45$ and/or the highest loadings on each component). Any components identified from the PCA were considered for subsequent analyses of EF differences among the groups.

Third, a MANOVA was performed using the EF components extracted from PCA, with group as a between-subject variable. We used mean regression factor scores (residuals extracted) from the PCA to compare group differences in the EF components; a constant (ie, 10) was added to the regression factor scores to eliminate negative values before MANOVA. For interpretability, composite scores for each significant factor were calculated and compared between the groups (eg, FLE versus controls and TLE versus controls) using MANOVA. A Bonferroni correction was used in all analyses.

Last, correlation analyses investigated associations between DTI indices and EF component scores, age at seizure onset, duration of epilepsy, and the number of AEDs.

\section{RESULTS}

\section{Delineation of Thalamocortical Pathways and Examination of Microstructure}

Left and right thalamocortical pathways were produced (Fig 1). Regardless of group, pathways were qualitatively identical (ie, they began and ended within similar neuroanatomic locations). On-line Table 2 contains means and SDs for each pathway measure and all multivariate and between-subject effects. The 
Table 2: Factor structure and factor loadings (>.45 and/or highest loading) after varimax rotation of 6 measures of EF in the FLE, TLE, and HC groups ( $n=55)$

\begin{tabular}{lcc}
\hline \multicolumn{1}{c}{ EF Measure } & EF1 & EF2 \\
\hline $\begin{array}{l}\text { D-KEFS Verbal Fluency Test: } \\
\text { Letter Fluency }\end{array}$ & - & .596 \\
$\begin{array}{l}\text { D-KEFS Verbal Fluency Test: } \\
\quad \text { Category Switching }\end{array}$ & - & .450 \\
$\begin{array}{l}\text { D-KEFS Color-Word Interference } \\
\quad \text { Test: Inhibition }\end{array}$ & .850 & - \\
$\begin{array}{l}\text { D-KEFS Color-Word Interference } \\
\quad \text { Test: Inhibition/Switching }\end{array}$ & .912 & - \\
$\begin{array}{l}\text { D-KEFS Sorting Test: Confirmed } \\
\quad \text { Correct Sorts }\end{array}$ & - & \\
CPT3 omission errors & & .824 \\
\hline
\end{tabular}

Note:-EFl indicates executive function component factor 1; EF2, executive function component factor 2

MANOVA yielded no significant effect of group for the left or right thalamocortical pathways.

\section{EF Components}

PCA extracted 2 latent components that were independent of each other and reflected independent aspects of EF. The KMO and Bartlett test validated that structure detection was appropriate for our data $\left[\mathrm{KMO}=0.742 ; \chi_{(15)}^{2}=88.25, P<.001\right]$. The component loadings for our 6 measures of EF are provided in Table 2. Together, these 2 components accounted for approximately $64 \%$ of the variance. The first component (EF1) explained 32.5\% of the variance in the data and reflected a mental flexibility/inhibition/ shifting dimension. Measures that loaded highly on this component included the D-KEFS Color-Word subtests. The highest loadings on the second component (EF2), explaining 31.2\% of the variance, reflected an attention/cognitive efficiency/problemsolving component of EF. Specifically, the CPT3 Omission Errors and D-KEFS Verbal Fluency and Sorting subtests were included in this dimension.

\section{Group Differences in EF}

When regression factor scores from the PCA were compared, we found group differences across both EF components $(\lambda=0.584$, $F=7.861, P<.001): \mathrm{EF} 1\left[F(2,52)=11.44, P<.001, \eta^{2}=0.306\right]$ and EF2 $\left[F(2,52)=3.426, P=.04, \eta^{2}=0.116\right]$. For EF1, the FLE group had scores significantly below HC and the TLE group $(P<$ .001 and $P=.007$, respectively). For EF2, the FLE group had scores significantly below $\mathrm{HC}(P=.04)$. No differences in EF component scores were found between the HC and TLE groups. For increased interpretability, composite factor scores were compared among the groups, yielding similar results as seen above (Fig 2).

When the FLE and TLE groups were divided on the basis of left-versus-right epileptogenic focus, we found group differences in the composite factor scores for both EF components $(\lambda=$ $0.511, F=4.692, P<.001): \mathrm{EF} 1[F(4,48)=7.218, P<.001]$ and $\mathrm{EF} 2[F(4,48)=7.173, P<.001]$. The left and right FLE groups had scores significantly below those of HC on both EF1 $(P=.001)$ and EF2 $(P<.001$ and $P=.009$, respectively) (Fig 3). Furthermore, the left FLE group had scores significantly below those of left TLE group $(P=.044)$. No differences in EF composite factor scores were found between the HC and TLE groups.

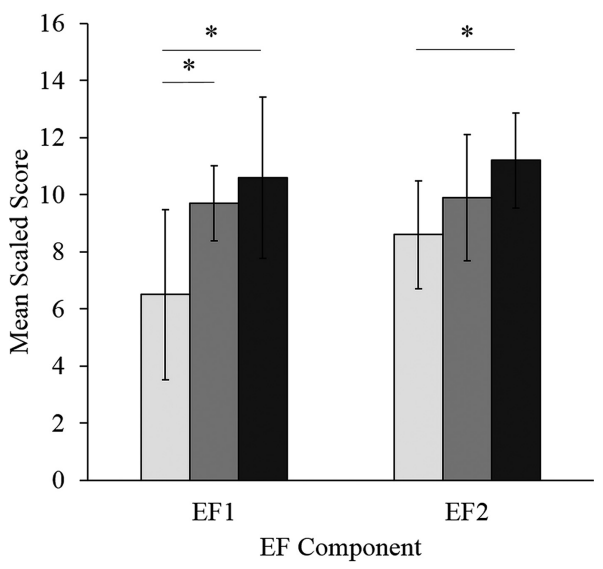

$\square$ FLE $\square$ TLE $\square \mathrm{HC}$

FIG 2. Mean composite EF factor scores for the FLE, TLE, and HC groups. Error bars represent SDs. The asterisk indicates a significant group difference (FLE $<\mathrm{HC}$ and FLE $<$ TLE for EFI and FLE $<\mathrm{HC}$ for EF2) at the $P<.05$ level. EFl represents a mental flexibility/inhibition/ switching component, while EF2 represents an attention/cognitive efficiency/problem-solving component.

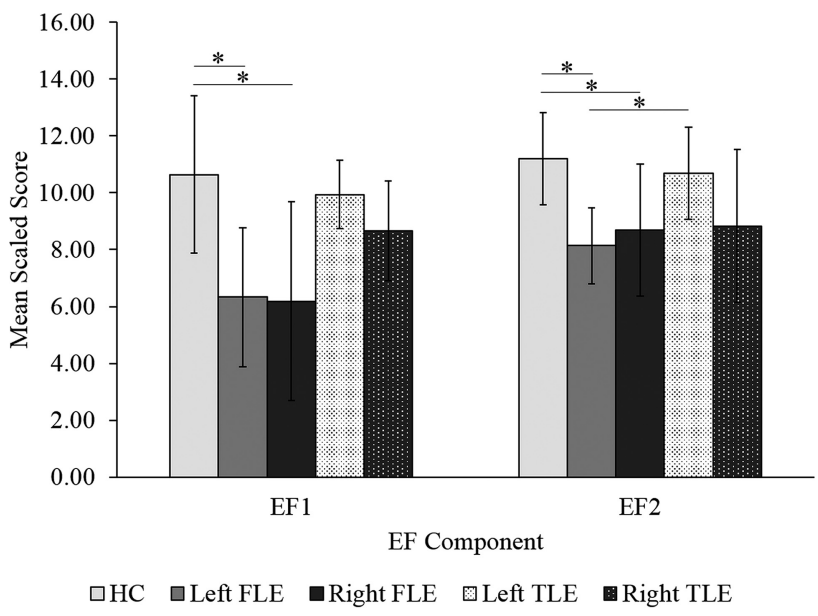

FIG 3. Mean composite EF scores for the left and right FLE, left and right TLE, and HC groups. Error bars represent SDs. The asterisk indicates a significant group difference (EFl: left FLE $<\mathrm{HC}$ and right FLE $<$ HC; EF2: left FLE $<\mathrm{HC}$, right FLE $<\mathrm{HC}$, left FLE $<$ left TLE) at the $P<$ .05 level. EFl represents a mental flexibility/inhibition/switching component, while EF2 represents an attention/cognitive efficiency/problem-solving component.

\section{EF and Thalamocortical Microstructure}

No correlations were found between EF1 or EF2 and the thalamocortical DTI indices for FLE group and HC. In the TLE group, EF1 was correlated with left thalamocortical RD $(r=-.559, P=$ $.047)$, while EF2 was correlated with left thalamocortical MD ( $r=$ $-.611, P=.026)$ and $\mathrm{RD}(r=-.620, P=.024)$, and with right thalamocortical RD $(r=-.566, P=.044)$.

\section{Age of Seizure Onset, Number of AEDs, Duration of Epilepsy, and Thalamocortical Microstructure}

In the FLE group, younger age at seizure onset was associated with lower fractional anisotropy and higher $\mathrm{MD}$ and $\mathrm{RD}$ of the thalamocortical pathways (left: $r=0.431, P=.035 ; r=-.412$, $P=.045 ; r=-.487, P=.016$, respectively; and right: $r=0.407$, $P=.049 ; r=-.530, P=.008 ; r=-.528, P=.008$, respectively).

In the FLE group, a greater number of AEDs was correlated 
with higher $\mathrm{MD}(r=0.491, P=.015)$ and $\mathrm{RD}(r=0.448, P=$ .028 ) of the right thalamocortical pathway. In the TLE group, there were no significant correlations between age of seizure onset or number of AEDs and measures of thalamocortical pathway microstructure. No correlations were found between the duration of epilepsy and any of the DTI indices for either group.

\section{DISCUSSION}

To our knowledge, the current study was the first to examine the white matter microstructure of circumscribed thalamocortical pathways in pediatric focal epilepsy using DTI. The investigation of structure-function relations in pediatric focal epilepsy gives insight into the impact of seizures on thalamocortical circuitry in the developing brain and consequently potential impairment in frontally mediated EF.

We found no differences in DTI indices of either the left or right thalamocortical pathway among children with left FLE, right FLE, left TLE, right TLE, and HC. These findings were somewhat surprising, given previous evidence of reduced frontal lobe connectivity in children with FLE. ${ }^{18-20}$ Our analyses were limited to connections between the thalamus and ipsilateral frontal cortex. Seizures may spread from the frontal or temporal lobe via white matter primarily to other lobes in the ipsilateral or contralateral hemisphere, rather than the thalamus. The current study did not examine structural measures of other frontal pathways and therefore may be missing areas that are injured in FLE or TLE. Furthermore, the thalamus is not the generator of seizures in FLE or TLE, which could, in part, explain the findings that the thalamocortical pathway was not significantly impaired in children with FLE or TLE. He et $\mathrm{al}^{33}$ found that thalamocortical functional connectivity to the parietal/occipital and temporal lobes was abnormal, but not thalamocortical connectivity to the frontal lobe in adults with TLE. Similarly, structural measures of thalamocortical architecture were documented to be no different between adults with idiopathic generalized epilepsy and healthy controls. ${ }^{7}$

Earlier age at FLE onset was associated with lower fractional anisotropy and higher MD and RD of the left and right thalamocortical pathways, implying that frontal lobe seizures early in life have a detrimental impact on thalamocortical connections. Specifically, FLE could interfere with normal development of thalamocortical pathways and, in turn, potentially affect function. We did not find associations between thalamocortical microstructure and the duration of epilepsy for either epilepsy group, suggesting that it may be the severity/extent of the epileptogenic activity, or early age at seizure onset, or both, not the cumulative time of epilepsy, which influence abnormal structure. These findings were like those in previous studies documenting a significant correlation between DTI measures and age of seizure onset but not duration of epilepsy. ${ }^{18,34}$

A greater number of AEDs in the FLE group was correlated with higher $\mathrm{MD}$ and $\mathrm{RD}$ of the right thalamocortical pathway. A higher number of AEDs may reflect greater seizure severity, which, in turn, may be associated with damaged or less developed white matter. Alternatively, it is possible that AEDs themselves may injure or impede developing white matter. ${ }^{35}$ However, it is difficult to tease apart the effects of seizure severity from the un- derlying contribution of AEDs on white matter development in this study.

Two latent components of EF were extracted from our data, reflecting the mental flexibility/inhibition/shifting dimension (EF1) and the attention/cognitive efficiency/problem-solving dimension (EF2). Children with left or right FLE had scores significantly below those of HC for EF1 and EF2. Our findings are in line with previous research in that difficulties with planning and impulse control, ${ }^{12,13,15}$ mental flexibility/set-shifting, ${ }^{13,17}$ attention, ${ }^{16}$ and verbal fluency ${ }^{13}$ have been documented in children with FLE relative to HC. Although difficulties with aspects of EF have been reported in TLE relative to controls, ${ }^{14}$ we did not observe differences in EF between TLE and HC, likely due to the relatively small sample sizes. Previous studies have revealed impairment in concept formation, ${ }^{17}$ mental flexibility/set-shifting, ${ }^{17,36}$ and attention/concentration ${ }^{36}$ in children with FLE compared with TLE. Congruently, we documented poorer performance on tasks that loaded onto EF2 (attention/cognitive efficiency/problem solving) for the FLE group compared with the TLE group; this difference was found only for children with left epileptogenic focus. The lack of a difference between the FLE and TLE groups for EF1 suggests that there may be a minimal or comparable impact of frontal and temporal lobe seizures on brain regions important for mental flexibility/inhibition/ shifting (EF1); the same may not be true for brain regions that underlie cognitive efficiency and problem solving (EF2), which is why we observed EF2 to be impacted in FLE.

Significant correlations between measures of thalamocortical microstructure and EF component scores emerged only for the TLE group. Specifically, higher scores on EF1 (the mental flexibility/inhibition/shifting) were correlated with lower RD of the left thalamocortical pathway. Furthermore, higher scores on EF2 (the attention/cognitive efficiency/problem solving) were associated with lower RD of the left and right thalamocortical pathways as well as lower MD of the left thalamocortical pathway. These findings suggest that greater insult or damage to the pathways (or less developed pathways) is associated with poorer scores on the EF1 in TLE. We expected to observe these findings for the FLE group because reduced connectivity within the frontal network has been related to performance on tasks of EF in children with FLE. ${ }^{18}$ Furthermore, cognitively impaired children with FLE showed the greatest white matter compromise throughout the brain, including decreased frontal lobe connectivity compared with cognitively unimpaired children with FLE and healthy controls. ${ }^{20}$ It is possible that an abnormal frontal lobe, and not solely thalamocortical pathways, is sufficient to cause EF impairment in FLE. However, for children with TLE, abnormal thalamocortical microstructure may be contributing to EF difficulties in the absence of overt frontal abnormality.

Limitations of the current study include the relatively small sample size, especially when considering whether laterality of seizure focus impacted both thalamocortical microstructure and EF outcomes. Furthermore, the reliability and validity of our PCA were limited by a relatively small overall sample size and, in turn, moderate-to-low participants-to-variables ratio (eg, 9:1). ${ }^{37}$ However, relatively high communalities $(>0.65)$ were found for all but 1 of our items, and many of our variables loaded strongly (ie, $>0.7$ ) and cleanly (ie, no cross-loadings; cutoff was loadings of 
$>0.45$ ) onto each factor (Table 2). Considering these findings, we are confident that our PCA findings can be considered valid.

Additionally, we acknowledge that several identified thalamocortical pathways exist (eg, the pulvinar and lateral geniculate nucleus of the thalamus and parietal-occipital cortex; ventral posterolateral, ventral posteromedial, and lateral posterior nuclei of the thalamus and somatosensory cortex). However, in terms of thalamofrontal connections, other than the anterior nuclei of the thalamus being linked with the prefrontal cortex, ${ }^{6}$ no other thalamic nuclei have been structurally associated with the frontal cortex using DTI tractography in children with epilepsy. We chose not to segment the thalamus to investigate specific connections between its nuclei and the frontal lobe for several reasons: Aside from small cell sizes across our groups, our FLE sample was a heterogeneous group in terms of the region of frontal epileptogenic focus (Table 1). It is possible that in choosing to delineate all possible connections between the thalamus and ipsilateral frontal lobe, we may be including fibers such as the anterior and posterior thalamic radiations; however, any fibers from the thalamus that fell outside the frontal lobe were not included in our analyses. Furthermore, we believed that the focus of initial investigations should be on examining "whole" thalamocortical connectionsthat is, all the potential connections between the thalamus and frontal lobe that met the specified threshold for connectivity. As a result, our study provides a basis for thalamocortical (ie, frontal) connectivity in the developing (and epileptogenic) brain.

Once these connections are identified in the developing brain, examined structurally, and linked to broad aspects of EF, then future research can begin to tease apart the microstructure of thalamocortical connections as they relate to function. For example, subdividing the thalamus into nuclei and investigating the corresponding cortical connections should be considered (eg, anterior or posterior thalamic radiations); contrasting these pathways in FLE and TLE may reveal microstructural differences. Additionally, the medial-dorsal nucleus of the thalamus has been implicated in the initiation and spread of seizures in TLE (thought to be related to reciprocal connections between the midline thalamus and the hippocampus, entorhinal cortex, amygdala, and the olfactory cortex complex). ${ }^{38}$ We did not include specific assessment of the thalamic medial dorsal nucleus because our patient population included children with FLE and TLE, and most of those with TLE had neocortical (or lateral) TLE rather than mesial TLE. Future research could include assessment of the connectivity between the medial dorsal thalamic nucleus and the hippocampus, entorhinal cortex, and amygdala in children with mesial TLE.

Last, although we investigated several EF tasks, further research is necessary to examine such functions as emotion regulation, planning, and organization in pediatric focal epilepsy populations and the potential impact of thalamocortical pathways on these aspects of EF. Because poorer performance on measures of EF was associated with thalamocortical microstructure in the TLE group, future research should investigate architectural differences in medial-versus-lateral TLE and implications on EF.

\section{CONCLUSIONS}

Our results suggest that in pediatric FLE, alterations to thalamocortical pathways at the structural level may not be sufficient to produce a functional deficit; the frontal cortex itself as well as other frontal-extrafrontal connections may play a significant role in EF impairment. Results from this study also give insight into potential mechanisms of EF impairment in pediatric TLE, in that thalamocortical pathway microstructure is associated with EF in children with TLE. Therefore, TLE may have a significant impact on extratemporal regions, including the frontal lobe and its connections. Identifying vulnerable neural systems in pediatric focal epilepsy and the relation of these systems and neurocognitive outcome will influence how we measure functional and cognitive/ behavioral outcome in this population.

Disclosures: Elysa Widjaja_UNRELATED: Grants/Grants Pending: Canadian Institutes of Health Research. * ${ }^{*}$ Money paid to the institution.

\section{REFERENCES}

1. Cavazos JE, Jones SM, Cross DJ. Sprouting and synaptic reorganization in the subiculum and CA1 region of the hippocampus in acute and chronic models of partial-onset epilepsy. Neuroscience 2004; 126:677-88 CrossRef Medline

2. Zhang CH, Sha Z, Mundahl J, et al. Thalamocortical relationship in epileptic patients with generalized spike and wave discharges: a multimodal neuroimaging study. Neuroimage Clin 2015;9:117-27 CrossRef Medline

3. Meeren HK, Pijn JP, Van Luijtelaar EL, et al. Cortical focus drives widespread corticothalamic networks during spontaneous absence seizures in rats. J Neurosci 2002;22:1480-95 CrossRef Medline

4. Castro-Alamancos MA. Neocortical synchronized oscillations induced by thalamic disinhibition in vivo. J Neurosci 1999;19:RC27 CrossRef Medline

5. Guye M, Regis J, Tamura M, et al. The role of corticothalamic coupling in human temporal lobe epilepsy. Brain 2006;129(Pt 7):1917-28 CrossRef

6. Deppe M, Kellinghaus C, Duning T, et al. Nerve fiber impairment of anterior thalamocortical circuitry in juvenile myoclonic epilepsy. Neurology 2008;71:1981-85 CrossRef Medline

7. McGill ML, Devinsky O, Wang X, et al. Functional neuroimaging abnormalities in idiopathic generalized epilepsy. Neuroimage Clin 2014;6:455-62 CrossRef Medline

8. Law N, Smith ML, Greenberg M, et al. Executive function in paediatric medulloblastoma: the role of cerebrocerebellar connections. J Neuropsychol 2017;11:174-200 CrossRef Medline

9. Fan Q, Davis N, Anderson AW, et al. Thalamo-cortical connectivity: what can diffusion tractography tell us about reading difficulties in children? Brain Connect 2014;4:428-39 CrossRef Medline

10. O’Muircheartaigh J, Vollmar C, Barker GJ, et al. Abnormal thalamocortical structural and functional connectivity in juvenile myoclonic epilepsy. Brain 2012;135:3635-44 CrossRef Medline

11. Alexander GE, Crutcher MD. Functional architecture of basal ganglia circuits: neural substrates of parallel processing. Trends Neurosci 1990;13:266-71 CrossRef Medline

12. Hernandez MT, Sauerwein HC, Jambaqué I, et al. Deficits in executive functions and motor coordination in children with frontal lobe epilepsy. Neuropsychologia 2002;40:384-400 CrossRef Medline

13. Luton LM, Burns TG, DeFilippis N. Frontal lobe epilepsy in children and adolescents: a preliminary neuropsychological assessment of executive function. Arch Clin Neuropsychol 2010;25:762-70 CrossRef Medline

14. Rzezak P, Fuentes D, Guimarães CA, et al. Frontal lobe dysfunction in children with temporal lobe epilepsy. Pediatr Neurol 2007;37: 176-85 CrossRef Medline

15. Culhane-Shelburne K, Chapieski L, Hiscock M, et al. Executive functions in children with frontal and temporal lobe epilepsy. I Int Neuropsychol Soc 2002;8:623-32 Medline

16. Hernandez MT, Sauerwein HC, Jambaqué I, et al. Attention, mem- 
ory, and behavioral adjustment in children with frontal lobe epilepsy. Epilepsy Behav 2003;4:522-36 CrossRef Medline

17. Longo CA, Kerr EN, Smith ML. Executive functioning in children with intractable frontal lobe or temporal lobe epilepsy. Epilepsy Behav 2013;26:102-08 CrossRef Medline

18. Widjaja E, Zamyadi M, Raybaud C, et al. Abnormal functional network connectivity among resting-state networks in children with frontal lobe epilepsy. AJNR Am J Neuroradiol 2013;34:2386-92 CrossRef Medline

19. Widjaja E, Kis A, Go C, et al. Bilateral white matter abnormality in children with frontal lobe epilepsy. Epilepsy Res 2014;108:289-94 CrossRef Medline

20. Braakman HM, Vaessen MJ, Jansen JF, et al. Frontal lobe connectivity and cognitive impairment in pediatric frontal lobe epilepsy. Epilepsia 2013;54:446-54 CrossRef Medline

21. Holt RL, Provenzale JM, Veerapandiyan A, et al. Structural connectivity of the frontal lobe in children with drug-resistant partial epilepsy. Epilepsy Behav 2011;21:65-70 CrossRef Medline

22. Campos BM, Coan AC, Beltramini GC, et al. White matter abnormalities associate with type and localization of focal epileptogenic lesions. Epilepsia 2015;56:125-32 CrossRef Medline

23. Liu Z, Xu Y, An J, et al. Altered brain white matter integrity in temporal lobe epilepsy: a TBSS study. J Neuroimaging 2015;25:460-64 CrossRef Medline

24. Paldino MJ, Hedges K, Zhang W. Independent contribution of individual white matter pathways to language function in pediatric epilepsy patients. Neuroimage Clin 2014;6:327-32 CrossRef Medline

25. Diao L, Yu H, Zheng J, et al. Abnormalities of the uncinate fasciculus correlate with executive dysfunction in patients with left temporal lobe epilepsy. Magn Reson Imaging 2015;33:544-50 CrossRef Medline

26. Koini M, Rombouts SA, Veer IM, et al. White matter microstructure of patients with neurofibromatosis type 1 and its relation to inhibitory control. Brain Imaging Behav 2017;11:1731-40 CrossRef Medline
27. Treit S, Chen Z, Rasmussen C, et al. White matter correlates of cognitive inhibition during development: a diffusion tensor imaging study. Neuroscience 2014;276:87-97 CrossRef Medline

28. Woolrich MW, Jbabdi S, Patenaude B, et al. Bayesian analysis of neuroimaging data in FSL. Neuroimage 2009;45:S173-86 CrossRef Medline

29. Behrens TE, Johansen-Berg H, Woolrich MW, et al. Non-invasive mapping of connections between human thalamus and cortex using diffusion imaging. Nat Neurosci 2003;6:750-57 CrossRef Medline

30. Woods RP, Grafton ST, Holmes CJ, et al. Automated image registration, I: general methods and intrasubject, intramodality validation. J Comput Assist Tomogr 1998;22:139-52 CrossRef Medline

31. Delis DC, Kaplan E, Kramer JH. Delis-Kaplan Executive Function System (D-KEFS). San Antonio: The Psychological Corporation; 2001

32. Conners KC. Conners' Continuous Performance Test. 3rd ed. Toronto: Multi-Health Systems; 2014

33. He X, Doucet GE, Sperling M, et al. Reduced thalamocortical functional connectivity in temporal lobe epilepsy. Epilepsia 2015;56: 1571-79 CrossRef Medline

34. Riley JD, Franklin DL, Choi V, et al. Altered white matter integrity in temporal lobe epilepsy: association with cognitive and clinical profiles. Epilepsia 2010;51:536-45 CrossRef Medline

35. Kaushal S, Tamer Z, Opoku F, et al. Anticonvulsant drug-induced cell death in the developing white matter of the rodent brain. Epilepsia 2016;57:727-34 CrossRef Medline

36. Riccio CA, Pliego JA, Cohen MJ, et al. Executive function performance for children with epilepsy localized to the frontal or temporal lobes. Appl Neuropsychol Child 2015;4:277-84 CrossRef Medline

37. Osborne JW, Costello AB. Sample size and subject to item ratio in principal components analysis. Practical Assessment, Research, \& Evaluation 2004;9:1-9

38. Bertram EH. Extratemporal lobe circuits in temporal lobe epilepsy. Epilepsy Behav 2014;38:13-18 CrossRef Medline 